J. Clin. Chem. Clin. Biochem.

Vol. 14, 1976, pp. 415-417

\title{
Erfahrungen mit der Glucose-Dehydrogenase-UV-Methode zur Bestimmung der Blutglucose
}

\author{
Von Roswitha Dolhofer, L. Weiss und O. H. Wieland \\ Klinisch-Chemisches Institut, Städt. Krankenhaus München-Schwabing, 8000 München 40, Kölner Platz 1
}

(Eingegangen am 12. März/23. Mä 1976)

Zusammenfassung: Eine Methode zur Blutzuckerbestimmung mit Glucose-Dehydrogenase wird beschrieben. Unserer Erfahrung mit dieser Methode liegen 70000 Blutzuckerbestimmungen innerhalb von sechs Monaten zu Grunde. Ein weiter Linearitätsbereich, Richtigkeit und Spezifität zeichnen diese Methode aus. Störungen durch physiologischerweise im Blut vorkommende Substanzen sind nicht bekannt. Die Glucose-Dehydrogenase-Methode korreliert gut mit der Hexokinase-Glucose-6-phosphatdehydrogenase-Methode.

\section{Experience with the Glucose-Dehydrogenase-UV-Method for the Determination of Blood Glucose}

Summary: A method for glucose determination using glucose-dehydrogenase is described. Our experience with this method is based on 70000 determinations of blood glucose within six months. Linearity over a wide range, accuracy and specificity highly recommend this method. No interference was noticed by substances physiologically occuring in blood. The glucose-dehydrogenase-method correlated well with the hexokinase glusoce-6-phosphatedehydrogenase method for the determination of blood glucose.

\section{Einführung}

Die Messung der Blutglucose gehört zu den häufigsten in einem klinisch-chemischen Laboratorium durchgeführten Untersuchungen. Eine Methode, die einfach und schnell durchzuführen ist, Spezifität und nur geringe Störanfälligkèit aufweist und darüber hinaus ökonomische Aspekte berücksichtigt, ist zu empfehlen. Eine von Banauch et al. (1) 1975 beschriebene Methode der Glucosebestimmung mit Glucose-Dehydrogenase (EC 1.1.1.47) aus Bacillus megaterium M 1286 wird diesen Ansprụ̈chen gerecht. Die vorliegende Arbeit berichtet über unsere Erfahrungen, die wir bei mehrmonatigem Einsatż dieser Methode im klinisch-chemischen Routinebetrieb gesammelt haben.

\section{Material und Methoden}

\section{Geräte}

1. Eppendorf Photometer $1100 \mathrm{M}$ mit Schreiber

2. Eppendorf Substratautomat 5030

3. Mikrolitersystem Eppendorf

\section{Reagenzien}

1. $30 \mathrm{~g} / \mathrm{l}(0,33 \mathrm{~mol} / \mathrm{l})$ Perchlorsäure

2. Testpackung „Merckotest Glucose" (Gluc-DH-Methode) UV-Test
3. Glucose-6-phosphat und Glucose-1-phosphat $\mathrm{Fa}$. Boehringer, Mannheim

4. Alle anderen Reagenzien stammten von der Fa. E. Merck, Darmstadt (Reinheitsgrad p.A. oder für biochemische Zwecke).

\section{Herstellung der Reagenzlösung}

Zur Glucosebestimmung werden die Enzyme Glucose-Dehydrogenase und Mutarotase in Natrium-Phosphatpuffer $\mathrm{pH} 7,6$, $0,12 \mathrm{~mol} / 1$ so gelöst, daß der Testansatz $5 \mathrm{kU} / 1$ Glucose-Dehydrogenase und 0,18 kU/1 Mutarotase (EC 5.1.3.3) enthält. Als Coenzym wird NAD in einer Endkonzentration von 2,2 mmol/1 der Pufferenzymlösung zugesetzt.

\section{Bestimmungsmaterial}

Für die Bestimmung kann Vollblut oder Plasma verwendet werden. Vollblut oder Plasma werden im Verhältnis 1:11 mit Perchlorsäure gemischt, 2 min bei etwa $10000 \times g$ zentrifugiert und der eiweißfreie Überstand zur Bestimmung eingesetzt.

\section{Manuelle Bestimmung}

$0,05 \mathrm{ml}$ Perchlorsäureüberstand werden mit $0,5 \mathrm{ml}$ Reagenzlösung $10 \mathrm{~min}$ bei $37^{\circ} \mathrm{C}$ (oder $15 \mathrm{~min}$ bei $25^{\circ} \mathrm{C}$ ) inkubiert und die Absorption der Probe gegen einen Reagenzienleerwert, bestehend aus $0,05 \mathrm{ml}$ Perchlorsäure und $0,5 \mathrm{ml}$ Reagenzlösung, bei $366 \mathrm{~nm}$ gemessen.

\section{Berechnung}

Die Berechnung der Glucosekonzentration erfolgt, sofern nicht anders angegeben, mittels des molaren Absorptionskoeffizienten für NADH, $\epsilon_{366}=3,3 \times 10^{6} \mathrm{~cm}^{2} / \mathrm{mol}$. Der Verdünnungsfaktor durch Perchlorsäure beträgt für Vollblut 10.85. 
Serienbestimmung am Analysenautomaten

Sämtliche Routinebestimmungen wurden am Eppendorf-Substratautomaten 5030 durchgeführt. Nach einer Inkubationszeit von $10 \mathrm{~min}$ bei $37^{\circ} \mathrm{C}$ erfolgte die Messung bei $366 \mathrm{~nm}$ gegen den Reagenzienleerwert. Die Reagenzien- und Probenvolumina entsprachen denen der manuellen Bestimmung.

\section{Ergebnisse und Diskussion}

Bei der Blutzuckerbestimmung mit Glucose-Dehydrogenase wird $\beta$-D-Glucose durch Glucose-Dehydrogenase zu Gluconsäurelacton dehydriert und dabei NAD zu NADH reduziert:

$\beta$-D-Glucose + NAD $\frac{\text { Glucose-Dehydrogenase }}{\text { nolacton }+\mathrm{NADH}+\mathrm{H}^{+}}$-Gluco-

Die Menge des gebildeten NADH ist der Glucosekonzentration direkt proportional und kann durch seine UVAbsorption photometrisch bestimmt werden. Da im Serum ein Gemisch aus $\alpha$-D.Glucóse und $\beta$ - $D$-Glucose vorliegt, die Glucose-Dehydrogenase jedoch nur die $\beta$-Form umzusetzen vermag, wird zur raschen Überführung der $\alpha$-Form in die $\beta$-Form dem Reagenz das Enzym Mutarotase zugesetzt.

$\alpha$-D-Glucose $\stackrel{\text { Mutarotase }}{=} \beta$-D-Glucose

Bei einer Reaktionstemperatur von $37^{\circ} \mathrm{C}$ wird bis zu einer Glucosekonzentration von 49,94 mmol/1 mit den angegebenen Enzymmengen nach 6 min ein konstanter Endwert erreicht.

\section{Linearität und Richtigkeit}

Die Linearität und Richtigkeit der Methode wurde mit eingewogenen Standards wasserfreier Glucose in Perchlorsäure überprüft. Es ergibt sich eine lineare Beziehung zwischen der NADH-Bildung und der eingesetzten Glucosemenge bis $49,94 \mathrm{mmol} / \mathrm{l}$. Als Regressionsgerade ergibt sich $y=0,991 x-0,334$, bei einem $r$ von 0,9999 $(y=$ gemessene Glucosemenge; $x$ = eingewogene Glucosemenge).

Die Wiederfindung von Glucose im Serum zeigt Tabelle 1. Angegeben ist der Mittelwert aus je 5 Einzelbestimmungen. Es decken sich erwarteter und gemessener Wert.

Tab. 1. Wiederfindungsrate von Glucose im Serum.

\begin{tabular}{lcrl}
\hline Serumkonzentration & Zugesetzt & \multicolumn{1}{l}{ Wiedergefunden } \\
\hline $12,87 \mathrm{mmol} / \mathrm{l}$ & $2,77 \mathrm{mmol} / \mathrm{l}$ & $2,89 \mathrm{mmol} / 1$ & $104 \%$ \\
$12,87 \mathrm{mmol} / \mathrm{l}$ & $5,16 \mathrm{mmol} / 1$ & $5,22 \mathrm{mmol} / 1$ & $101 \%$ \\
$12,87 \mathrm{mmol} / 1$ & $10,65 \mathrm{mmol} / 1$ & $10,54 \mathrm{mmol} / \mathrm{l}$ & $99 \%$ \\
$12,87 \mathrm{mmol} / 1$ & $24,97 \mathrm{mmol} / 1$ & $24,52 \mathrm{mmol} / 1$ & $98 \%$ \\
\hline
\end{tabular}

\section{Qualitätskontrolle}

Die Präzision in der Serie, erstellt am Eppendorf-Automaten, ergab bei einer Konzentration von 5,55 mmol/1 einen Variationskoeffizienten von 2,1\%, bei 27,75 $\mathrm{mmol} / \mathrm{l}$ einen von $0,5 \%$. Die Präzision von Tag zu Tag, wurde mit dem Kontrollserrum ,Ledertrol-U“ (Charge 2908-G $24 \mathrm{~K} 3)(\overline{\mathrm{x}}=15,26 \mathrm{mmol} / \mathrm{l})$ über ein halbes Jahr bestimmt. Der Variationskoeffizient schwankte monatlich von $1,3-2,4 \%$.

Für die kommerziellen Kontrollseren waren zum Zeitpunkt unserer Messungen keine Sollwerte für die Glucose-Dehydrogenase-UV-Methode ermittelt. Das Ergebnis im Vergleich zu den gegenwärtigen Standardmethoden, der Hexokinase-Glucose-6-phosphatdehydrogenaseMethode (2) zeigt Tabelle 2. Angegeben sind jeweils die Mittelwerte aus Fünffachbestimmungen. Die Werte liegen im Durchschnitt niedriger als die für die Hexokinase-Glucose-6-phosphatdehydrogenase-Methode ermittelten Werte, und entsprechen eher den Sollwerten für die Glucose-Oxidase-Perid-Methode.

Tab. 2. Analyse von Kontroliseren.

\begin{tabular}{|c|c|c|c|}
\hline \multirow[b]{2}{*}{ Kontrollserum } & \multicolumn{2}{|c|}{ Sollwerte } & \multirow[b]{2}{*}{$\begin{array}{l}\text { Glucose- } \\
\text { Dehydro- } \\
\text { genase-UV- } \\
\text { Methode }\end{array}$} \\
\hline & $\begin{array}{l}\text { Hexo- } \\
\text { kinase- } \\
\text { Methode }\end{array}$ & $\begin{array}{l}\text { Glucose- } \\
\text { Oxidase- } \\
\text { Perid- } \\
\text { Methode }\end{array}$ & \\
\hline & $\mathrm{mmol} / 1$ & $\mathrm{mmol} / \mathrm{l}$ & $\mathrm{mmol} / \mathrm{l}$ \\
\hline 1) & & & \\
\hline $\begin{array}{l}\text { Normosic Nr. } 405 \\
\text { 2) }\end{array}$ & 3,33 & 2,94 & 2,89 \\
\hline $\begin{array}{l}\text { Kontrollogen L } \\
\text { Nr. } 415\end{array}$ & 4,44 & 4,34 & 4,16 \\
\hline $\begin{array}{l}\text { 3) } \\
\text { Seronorm Nr. } 126\end{array}$ & 7,49 & 7,16 & 6,99 \\
\hline 3) & - & 1,94 & 1,89 \\
\hline 3) & $\therefore$ & 14,37 & 13,87 \\
\hline $\begin{array}{l}\text { 4) } \\
\text { Monitrol II Nr. } 32\end{array}$ & 12,76 & 12,93 & 12,76 \\
\hline $\begin{array}{l}\text { 5) } \\
\text { Precipath S Nr. } 302\end{array}$ & 17,87 & 18,31 & 16,65 \\
\hline
\end{tabular}

1) Asid, 2) Behring, 3) Molter, 4) Dade, 5) Boehringer.

\section{Probenleerwert}

Um abzuklären, ob bei dieser Methode auf einen Probenleerwert verzichtet werden kann, wurden Perchlorsäureüberstände von 136 Blutproben mit NAD-freier Reagenzlösung inkubiert und gegen den Reagenzienleerwert (s. „Manuelle Bestimmung“) gemessen. Die durchschnittliche Absorptionsdifferenz lag bei 0,003 , entsprechend einem Glucosegehalt von $0,1 \mathrm{mmol} / 1$ (maximal 0,27 mmol/1), so daß im Routinebetrieb auf einen Probenleerwert verzichtet werden kann. Werden jedoch die mit Perchlorsäure enteiweißten Blutproben nur mit etwa $3000 \times g$ zentrifugiert, so werden ohne Probenleerwert- 
korrektur durch Resttrübung im Mittel um 0,85 mmol/1 (maximal bis zu 1,66 mmol/l) überhöhte Blutglucosewerte vorgetäuscht.

\section{Stabilität des Reagenzgemisches}

Zur Prüfung der Stabilität des Reagenzgemisches wurde dieses bei Raumtemperatur aufbewahrt und in 3-tägigen Abständen durch Bestimmung von Standards von $2,77 \mathrm{mmol} / 1,22,19 \mathrm{mmol} / 1$ und $41,62 \mathrm{mmol} / 1$ überprüft. Über den geprüften Zeitraum von 8 Wochen blieb die Reagenzlösung stabil und erlaubte die exakte Bestimmung der Glucosestandards.

\section{Methodenvergleich}

An 325 Blutproben wurden Vergleichsuntersuchungen mit der Hexokinase-Glucose-6-phosphatdehydrogenaseMethode $(2,4)$ durchgeführt. Beide Meßreihen wurden mit dem Eppendorf-Substratautomaten erstellt. Der Korrelationskoeffizient von $\mathrm{r}=0,9951$ zeigt eine gute Uber-

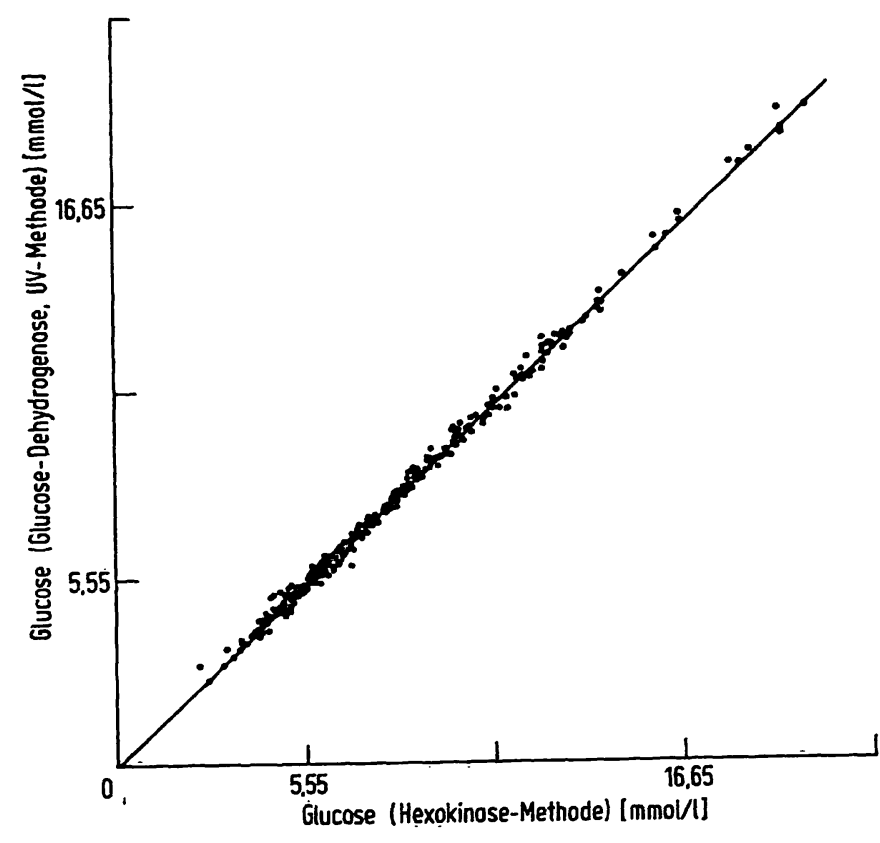

Abb. 1. Vergleich żwischen Hexokinase-Methode (x) und Glucose-Dehydrogenase-UV-Methode (y). einstimmung zwischen beiden Bestimmungen. Als Regressionsgerade findet man $: y=0,969 x-0,452$. Die mit der Glucose-Dehydrogenase-Methode erhaltenen Werte liegen also durchschnittlich um 3,5\% niedriger als die mit der Hexokinase-Methode erhaltenen (Abb. 1). Diese konstante Abweichung ergab sich jedoch nur, wenn in beiden Methoden mit dem bisher noch gebräuchlichen Absorptionskoeffizienten von $\epsilon_{366}=3,3 \times 10^{6} \mathrm{~cm}^{2} / \mathrm{mol}$ gerechnet wurde. Benützt man für NADPH den Wert $\epsilon_{365}=3,5 \times 10^{6} \mathrm{~cm}^{2} / \mathrm{mol}$ und für NADH $\epsilon_{365}=3,4 \times 10^{6}$ $\mathrm{cm}^{2} / \mathrm{mol}$ (5), so findet man nahezu identische Werte. Bei einem $\mathrm{r}=0,9988$ wird $\mathrm{y}=1,002 \mathrm{x}-1,289$.

\section{Spezifität und Störungen}

Zur Prüfung der Spezifität der Glucose-DehydrogenaseMethode wurden anstelle von Glucose folgende Substanzen als Substrate in einer Konzentration von 100 $\mathrm{mg} / \mathrm{dl}$ zur Bestimmung eingesetzt: Fructose, Galaktose, Mannose, Ribose, Desoxyribose, Xylose, Lactose, Glucose-1-P, Glucose-6-P. Meßbare Absorptionen wurden nur mit Xylose und Mannose erhalten, nämlich 16\% bzw. 3\% der Absorption einer Glucoselösung gleicher Konzentration. Da die Konzentration für Mannose im Blut von Normalpersonen bei $55,5 \mu \mathrm{mol} / \mathrm{l}$ (6) liegt, ist dieser Wert zu vernachlässigen. Die Xylose-Konzentration im Normalblut liegt bei etwa $165 \mu \mathrm{mol} / \mathrm{l}$ (7), so daß auch hier keine Störungen zu erwarten sind. Nach oraler Xylose-Belastung werden jedoch Blutspiegel erreicht, die mit der Glucose-Dehydrogenase-Methode zu fälschlich erhöhten Glucosewerten führen.

Bilirubin $342 \mu \mathrm{mol} / \mathrm{l}$, Harnsäure $1190 \mu \mathrm{mol} / 1$, Kreatinin $2211 \mu \mathrm{mol} / \mathrm{l}$, Ascorbinsäure $11,36 \mathrm{mmol} / \mathrm{l}$, Glutathion $651 \mu \mathrm{mol} / \mathrm{l}(1)$ und Antikoagulantien (1) beeinflussen die Methode nicht.

Bei bisher etwa 70000 durchgeführten Bestimmungen erwies sich die beschriebene Methode als spezifisch, störunanfällig, schnell und einfach durchführbar. Die große Reagenzienstabilität, der weite Linearitätsbereich und nicht zuletzt die Wirtschaftlichkeit gehören zu den weiteren Vorzügen der Glucose-Dehydrogenase-UVMethode.

\section{Literatur}

1. Banauch, D,; Brunner, W., Ebeling, W., Metz, H., Rindfrey, H. \& Lang, H. (1975), diese Z. 13,101-107.

2 Bergmeyer, H. U., Bernt, E., Schmidt, F. \& Stork, H. (1974). In: Methoden der enzymatischen Analyse. (Bergmeyer, H. U., Ëd.) 3. Aufl, pp. 1241-1246, Verlag Chemie, Weinheim/ Bergstraße.

3. Bergmeyer, H. U. \& Bernt, E. (1974). In: Methoden der enzymatischen Analyse. (Bergmeyer, H. U., Ed.) 3. Aufl., pp. 1250 -1259 , Verlag Chemie, Weinheim/Bergstraße.
4. Weiss, L. In: Laboratoriumsdiagnose akuter Syndrome. Thieme Stuttgart, in Druck.

5. Bergmeyer, H. U. (1975), diese Z. 13, 507-508.

6. Jolley, R. L., Warren, K. S., Scott, C. D., Jainchill. J. C.

\& Freeman, M. L. (1970). J. Clin. Pathol. 53, 793-802.

7. Hawkins, K. J. (1970). Clin. Chem. 16, 749-752.

8. Müller-Matthesius, R. (1975), diese Z 13,169-170.

9. Müller-Matthesius, R. (1975), diese Z. 13,187-189.

Dr. Roswitha Dolhofer Städt. Krankenhaus München-Schwabing Klinisch-chemisches Institut Kölner Platz 1 8000 München 40 